\title{
Geometric BRST Quantization, I: Prequantization
}

\author{
José M. Figueroa-O'Farrill ${ }^{1}$, and Takashi Kimura ${ }^{2 \star \star}$ \\ 1 Instituut voor Theoretische Fysica, Universiteit Leuven, Celestijnenlaan 200D, B-3001 Heverlee, \\ Belgium \\ 2 Department of Mathematics, 61200 University of Texas, Austin, Texas 78712, USA
}

Received September 15, 1989

\begin{abstract}
This is the first part of a two-part paper dedicated to the definition of BRST quantization in the framework of geometric quantization. After recognizing prequantization as a manifestation of the Poisson module structure of the sections of the prequantum line bundle, we define BRST prequantization and show that it is the homological analog of the symplectic reduction of prequantum data. We define a prequantum BRST cohomology theory and interpret it in terms of geometric objects. We then show that all Poisson structures correspond under homological reduction. This allows to prove, in the BRST context, that prequantization and reduction commute.
\end{abstract}

\section{Introduction}

This is the first part of a two-part paper aimed at defining BRST quantization. Although BRSTquantization has become the preferred method to quantize gauge systems, almost no attention has been focused on the problem of defining this procedure formally nor to justify its validity; the only justification for the validity of the BRST quantization procedure being that it is analogous to its betterunderstood classical counterpart. The BRST quantization of a gauge theory consists roughly in the quantization of a larger system to which it corresponds classically (after homological reduction). However only in very special systems (e.g., free string theory) can one actually show that the quantum theories also correspond (after homological reduction).

We shall work throughout this paper in the symplectic or hamiltonian framework in which classical BRST appears in its more natural form. To fix the ideas, let $(M, \Omega)$ be a symplectic manifold in which one has defined a set of irreducible first class constraints. Then there is a geometric construction (outlined in Sect. 2)

\footnotetext{
* BITNET: fgbda11@blekul11

$\star \star$ Internet: kimura@math.utexas.edu
} 
by which - if the constraints are sufficiently nice - one can reduce $(M, \Omega)$ to a lower-dimensional symplectic manifold $(\tilde{M}, \tilde{\Omega})$. This geometric construction, called symplectic reduction, provides the geometric underspinning for the classical BRST procedure; or, equivalently, classical BRST provides an algebraic (more precisely, homological) realization of symplectic reduction.

The quantization of constained systems consists in the successful completion of the following diagram:

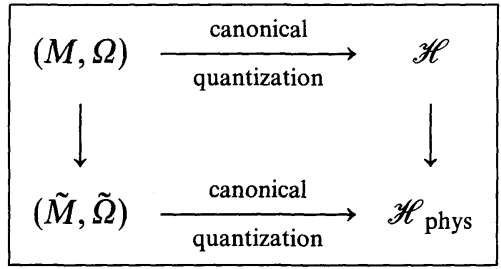

where by $\mathscr{H}$ and $\mathscr{H}_{\text {phys }}$ we mean the hilbert spaces of the respective quantum theories and by successful we mean that the rightmost arrow can be constructed in such a way that the diagram commutes. That is, to go from the initial data $(M, \Omega)$ to the physically meaningful quantum theory on $\mathscr{H}_{\text {phys }}$ it does not matter which route we take: we could either first symplectically reduce $(M, \Omega)$ to $(\tilde{M}, \tilde{\Omega})$ and then canonically quantize this latter symplectic manifold, or we would quantize $(M, \Omega)$ directly and then recover algebraically the physical states ${ }^{1}$. In practice the second route is preferred because either the symplectic reduction is hard to do explicitly or, even when it can be done, one may lose desirable properties of the redundant formalism: locality, covariance, ... . Nevertheless even when in practice there is only one way to effectively construct the quantum theory it is important to verify that the diagram is commutative. The only results of a general nature that hint at the commutativity of (1.1) are the results of Guillemin and Sternberg [1] which essentially state its commutativity for the special case of $M$ a (simply connected) compact Kähler manifold and $\tilde{M}$ its reduction via the Poisson action of a (simply connected) compact Lie group. However, the conjecture of commutativity is further supported by our experience with certain exactly solvable systems, for instance, free string theory where we can do BRST quantization or go to the lightcone, and both theories have been shown to have the same spectrum. See also the recent article by Duval, Elhadad, and Tuynman [2], where the commutativity of the diagram is shown to hold for some special systems. In this first part of the paper we prove the commutativity of the related diagram to (1.1) in which the horizontal arrows correspond to prequantization: the first step in the geometric quantization program.

In defining BRST quantization it seems natural to exploit the symplectic nature of classical BRST. There are two quantization prescriptions which can be defined intrinsically in terms of symplectic data: geometric quantization and Poisson deformations. We will focus only in the former method; leaving the Poisson deformation formulation of BRST quantization to a further publication.

\footnotetext{
${ }^{1}$ Matters are further complicated by the fact that the horizontal arrows in (1.1) do not exist in general. This is nothing but the statement that given a classical dynamical system there is no unique way to quantize it, or sometimes it cannot be quantized at all ("first quantization is not a functor," "there is nothing canonical about canonical quantization"); but we should not let this thwart our plans
} 
Roughly speaking quantization consists of finding a representation for the Lie algebra of real valued smooth functions on a symplectic manifold as self-adjoint operators in a Hilbert space subject to a finite reducibility condition forced by the uncertainty relation. It is well known that such a representation does not exist: either we relax the finite reducibility condition or we restrict the range of the representation to a subalgebra of the smooth functions. Geometric quantization is an attempt at developing a mathematically consistent quantization scheme. It consists of two steps: prequantization and polarization. In prequantization the finite reducibility condition is relaxed and one simply constructs the representation. Then polarization takes care of restricting the Hilbert space and in the process the functions that can be represented. The two steps are fairly independent and therefore we treat only prequantization in this paper. In the second part of this paper [3] we will discuss polarizations.

This paper is organized as follows. In Sect. 2 we briefly review the main facts of classical BRST that we need in the sequel. We have tried to be as brief as possible while still maintaining a reasonable level of comprehensiveness. Consequently no proofs are given and we have limited ourselves to introducing the objects and the main results. The section starts with a brief discussion of symplectic reduction which, aside from being the geometric motivation behind classical BRST, is a good picture to keep in mind throughout the algebraic constructions which follow. The section then continues with a brief description of classical BRST from the point of view that it is the algebraic realization of a topological subquotient. Finally the section concludes with a description of the Poisson structure of classical BRST: a structure which plays a fundamental rôle in all our considerations. In Sect. 3 we introduce prequantization and show that it is intimately related to the notion of a Poisson module. We have not seen Poisson modules defined anywhere ${ }^{2}$ but we feel our definition is the natural one. Reasoning by analogy we then define a prequantum BRST operator. In Sect. 4 the cohomology of the prequantum BRST operator is analyzed. We employ a spectral sequence argument together with some bundle gymnastics to relate it to a certain cohomology theory of vertical forms with coefficients in a line bundle. In order to obtain these results we need a purely algebraic lemma which is relegated to the appendix although promoted to a theorem. We then show that the zeroth dimensional prequantum BRST cohomology corresponds to the sections of a prequantum line bundle over the reduced symplectic manifold as we expected. After the construction of an invariant pointwise inner product we are able to show that all of the prequantum data gets induced via BRST. In other words, we show that prequantization and reduction commute in general. Finally in Sect. 5 we offer some concluding remarks.

\section{Classical BRST}

In this section we will review the classical BRST construction. Since the motivation for the construction lies in symplectic reduction we briefly review this in the first subsection. The next three subsections outline the BRST construction. The final subsection places the BRST construction within the framework on Poisson superalgebras.

\footnotetext{
2 Although in a very interesting recent paper by Huebschmann [4] they are seen to be special cases of a modules over a much more general kind of algebra than Poisson algebras: Rinehart's $(A, R)$-algebras
} 


\section{Symplectic Reduction}

Let us start by reviewing the reduction of a symplectic manifold by a coisotropic submanifold. In particular we are interested in the case where the submanifold is the zero locus of a set of first class constraints. The interested reader can consult [5] for a very readable exposition of this subject.

Let $(M, \Omega)$ be a $2 n$-dimensional symplectic manifold and $i: M_{0} \rightarrow M$ a coisotropic submanifold. That is, for all $m \in M_{0}, T_{m} M_{0}^{\perp} \subseteq T_{m} M_{0}$, where $T_{m} M_{0}^{\perp}=$ $\left\{X \in T_{m} M \mid \Omega(X, Y)=0 \forall Y \in T_{m} M_{0}\right\}$. Because $d \Omega=0$, the distribution $m \mapsto T_{m} M_{0}^{\perp}$ is involutive and it is known as the characteristic or null distribution of $i^{*} \Omega$. If its dimension is constant then, by Frobenius theorem, $M_{0}$ is foliated by maximal connected submanifolds having the characteristic distribution as its tangent space. There is a natural surjective map $\pi$ from $M_{0}$ to the space of leaves $\tilde{M}$ sending each point in $M_{0}$ to the unique leaf containing it. If the foliation is fibrating the space of leaves inherits a smooth structure making $\pi$ a smooth surjection. If that is the case there is a symplectic structure $\tilde{\Omega}$ on $\tilde{M}$ obeying $\pi^{*} \tilde{\Omega}=i^{*} \Omega$. The resulting symplecting manifold $(\tilde{M}, \tilde{\Omega})$ is called the symplectic reduction of $M$ by $M_{0}$.

In this paper we will focus on a very specific kind of coisotropic submanifold. Let $\left\{\phi_{i}\right\}$ be a set of $k$ smooth functions (constraints) on $M$ and let $J$ denote the ideal they generate in $C^{\infty}(M)$. The constraints are said to the first class if $J$ is a Lie subalgebra of $C^{\infty}(M)$ under Poisson bracket. This is clearly equivalent to the existence of smooth functions $\left\{f_{i j}{ }^{k}\right\}$ such that $\left\{\phi_{i}, \phi_{j}\right\}=\sum_{k} f_{i j}{ }^{k} \phi_{k}$. Assembling the $\phi_{i}$ together into one smooth function $\Phi: M \rightarrow \mathbb{R}^{k}$, we define $M_{0} \equiv \Phi^{-1}(0)$. If 0 is a regular value of $\Phi$ - i.e., the tangent map $d \Phi$ is surjective - the constraints are called regular. In this case $M_{0}$ is a closed embedded coisotropic submanifold of $M$. Let $X_{i}$ denote the hamiltonian vector field associated to $\phi_{i}$. The characteristic distribution of $M_{0}$ is spanned by the $\left\{X_{i}\right\}$. If the $\left\{\phi_{i}\right\}$ are constraints of a dynamical system whose phase space is $M$ then the leaves of the foliation determined by the $\left\{X_{i}\right\}$ are the "gauge orbits" and if the foliation fibers the space of orbits $\tilde{M}$ is the reduced phase space.

A very important special case of symplectic reduction comes about when the constraints arise from a hamiltonian group action [6]. Let $G$ be a connected Lie group acting on $M$ via symplectomorphisms. To each element $X$ in the Lie algebra $\mathfrak{g}$ of $G$ we associate a Killing vector field $\tilde{X}$ on $M$ which is symplectic. To each symplectic vector field there is associated a closed 1-form on $M: i(\tilde{X}) \Omega$. If this form is exact then the vector field is hamiltonian. If all the Killing vector fields are hamiltonian the $G$-action is called hamiltonian. In this case we can associate to each vector $X \in \mathfrak{g}$ a hamiltonian functions $\phi_{X}$ such that $d \phi_{X}+i(\tilde{X}) \Omega=0$. Dual to this construction is the moment map $\Phi: m \rightarrow \mathrm{g}^{*}$ defined by $\langle\Phi(m), X\rangle=\phi_{X}(m)$, for all $m \in M$. If a certain cohomology obstruction is overcome the hamiltonian functions $\left\{\phi_{X}\right\}$ close under Poisson bracket: $\left\{\phi_{X}, \phi_{Y}\right\}=\phi_{[X, Y]}$. If this is the case, the moment map is equivariant: intertwining between the $G$-actions on $M$ and the coadjoint action on $\mathrm{g}^{*}$.

Assume that we have a hamiltonian $G$-action on $M$ giving rise to an equivariant moment map and furthermore suppose that $0 \in \mathrm{g}^{*}$ is a regular value of the moment map. Denote $\Phi^{-1}(0)$ by $M_{0}$. Then $M_{0}$ is a closed embedded coisotropic submanifold and for $m \in M_{0}, T_{m} M_{0}^{\perp}$ is precisely the subspace spanned by the Killing vectors. In this case the leaves of the foliation are just the orbits of the 
$G$-action. If the $G$-action on $M_{0}$ is free and proper then the space of orbits inherits the structure of a smooth symplectic manifold in the way described above. If the $G$-action on $M_{0}$ is not free but only locally free (i.e., the isotropy is discrete) then the space of orbits is a symplectic orbifold.

It is interesting to notice that the reduced symplectic manifold is always a subquotient of $M$. That is, first we restrict to a submanifold $\left(M_{0}\right)$ and then we project onto the space of leaves of the null foliation. This is to be compared with cohomology which is also a subquotient of the cochains: first we restrict to the subspace of the cocycles and then we project by factoring out the coboundaries. It is therefore not surprising that one can set up a cohomology theory on $M$ which recovers the symplectic quotient $\tilde{M}$; or, rather, which recovers $C^{\infty}(\tilde{M})$ from $C^{\infty}(M)$. This is precisely what the classical BRST cohomology achieves [7,8]. In fact, as we will see classical BRST consists of three different steps. The first two take care of providing homological analogs for the passage from $M$ to $M_{0}$ and for the passage from $M_{0}$ to $\tilde{M}$. The final step integrates the first two into one homomology theory: BRST.

\section{Restriction: from $M$ to $M_{0}$}

We first focus on the restriction part of symplectic reduction: the passage from $M$ to $M_{0}$; or, in our more algebraic description, from $C^{\infty}(M)$ to $C^{\infty}\left(M_{0}\right)$. Since $M_{0}$ is a closed embedded submanifold of $M$, any smooth function on $M_{0}$ extends to a smooth function on $M$ and the difference of any two such extensions vanishes on $M_{0}$. Hence if we let $I\left(M_{0}\right)$ denote the (multiplicative) ideal of $C^{\infty}(M)$ consisting of functions which vanish at $M_{0}$, we have the following isomorphism

$$
C^{\infty}\left(M_{0}\right) \cong C^{\infty}(M) / I\left(M_{0}\right),
$$

where, moreover, since the constraints are regular, $I\left(M_{0}\right)$ is precisely the ideal $J$ generated by the constraints. However, we would rather work with the constraints themselves than with ideal they generate. The solution to this problem relies on a construction due to Koszul. There is a differential complex (the Koszul complex)

$$
\ldots \rightarrow K^{2} \rightarrow K^{1} \rightarrow C^{\infty}(M) \rightarrow 0,
$$

whose homology in positive dimensions is zero and in zero dimension is precisely $C^{\infty}\left(M_{0}\right)$ : a fact we shall refer to as the "quasi-acyclicity" of the Koszul complex. This fact plays a fundamental rôle in all our constructions.

It is convenient to describe the Koszul complex in more generality. Let $R$ be a (commutative, unital) ring and let $\Phi=\left(\phi_{1}, \ldots, \phi_{k}\right)$ be a sequence of elements of $R$. The immediate example we have in mind is $R=C^{\infty}(M)$ and $\left\{\phi_{i}\right\}$ the first class constraints. A sequence $\left(\phi_{1}, \ldots, \phi_{k}\right)$ of elements of $R$ is called regular if for all $j=1, \ldots, k, \phi_{j}$ is not a zero divisor in $R / I_{j-1}$, where $I_{j}$ is the ideal generated by $\phi_{1}, \ldots, \phi_{j}$ and $I_{0}=0$. In other words, if $f \in R$ and for any $j=1, \ldots, k$, $\phi_{j} f \in I_{j-1}$ than $f \in I_{j-1}$ to start out with. If the constraints are regular (in the differential geometric sense) then the sequence they define in $C^{\infty}(M)$ is regular (in the algebraic sense).

We define the Koszul complex $K(\Phi)$ as follows: $K^{0}(\Phi)=R$ and for $p>0$, $K^{p}(\Phi)$ is defined to be the free $R$ module with basis $\left\{e_{i_{1}} \wedge \ldots \wedge e_{i_{p}} \mid 0<i_{1}<\right.$ $\left.\ldots<i_{p} \leq k\right\}$. Define a map $\delta_{K}: K^{p}(\Phi) \rightarrow K^{p-1}(\Phi)$ by $\delta_{K} e_{i}=\phi_{i}$ and extending 
to all of $K(\Phi)$ as an $R$-linear antiderivation. That is, $\delta_{K}$ is identically zero on $K^{0}(\Phi)$ and

$$
\delta_{K}\left(e_{i_{1}} \wedge \ldots \wedge e_{i_{p}}\right)=\sum_{j=1}^{p}(-1)^{j-1} \phi_{i_{j}} e_{i_{1}} \wedge \ldots \wedge \widehat{e_{i_{j}}} \wedge \ldots \wedge e_{i_{p}},
$$

where a adorning a symbol denotes its omission. It is trivial to verify that $\delta_{K}^{2}=0$, yielding a complex

$$
0 \rightarrow K^{k}(\Phi) \stackrel{\delta_{K}}{\longrightarrow} K^{k-1}(\Phi) \rightarrow \ldots \rightarrow K^{1}(\Phi) \rightarrow R \rightarrow 0 .
$$

The following theorem is a classical result in homological algebra whose proof is completely straightforward and can be found, for example, in [9].

Theorem 2.5. If $\left(\phi_{1}, \ldots, \phi_{k}\right)$ is a regular sequence in $R$ then the homology of the Koszul complex is given by

$$
H^{p}(K(\Phi)) \cong\left\{\begin{array}{lll}
0 & \text { for } & p>0 \\
R / J & \text { for } & p=0
\end{array}\right.
$$

where $J$ is the ideal generated by the $\phi_{i}$.

Therefore the complex $K(\Phi)$ provides an acyclic resolution (known as the Koszul resolution) for the $R$-module $R / J$. This yields the following immediate corollary:

Corollary 2.7. If 0 is a regular value for $\Phi: M \rightarrow \mathbb{R}^{k}$ the Koszul complex $K(\Phi)$ gives an acyclic resolution for $C^{\infty}\left(M_{0}\right)$. In other words, the cohomology of the Koszul complex is given by

$$
H^{p}(K(\Phi)) \cong\left\{\begin{array}{lll}
0 & \text { for } & p>0 \\
C^{\infty}\left(M_{0}\right) & \text { for } & p=0
\end{array}\right.
$$

where $M_{0} \equiv \Phi^{-1}(0)$

\section{Quotient: from $M_{0}$ to $\tilde{M}$}

Having taken care of the restriction process we now focus on the second part of the classical BRST construction: the passage from $C^{\infty}\left(M_{0}\right)$ to $C^{\infty}(\tilde{M})$.

A smooth function on $\tilde{M}$ pulls back to a smooth function on $M_{0}$ which is constant on the fibers. Conversely, any smooth function on $M_{0}$ which is constant on the fibers defines a smooth function on $\tilde{M}$. Since the fibers are connected (after all they arise as integral submanifolds of a distribution) a function is constant on the fibers if and only if it is locally constant. Since the hamiltonian vector fields $\left\{X_{i}\right\}$ associated to the constraints $\left\{\phi_{i}\right\}$ form a global basis of the tangent space to the fibers, a function $f$ on $M_{0}$ is locally constant on the fibers if and only if $X_{i} f=0$ for all $i$. In an effort to build a cohomology theory and in analogy to the de Rham theory, we pick a global basis $\left\{\omega^{i}\right\}$ for the cotangent space to the fibers such that they are dual to the $\left\{X_{i}\right\}$, i.e., $\omega^{i}\left(X_{j}\right)=\delta_{j}^{i}$. We then define the vertical derivative $d_{V}$ on functions as

$$
d_{V} F=\sum_{i}\left(X_{i} f\right) \omega^{i} \quad \forall f \in C^{\infty}\left(M_{0}\right)
$$


Let $\Omega_{V}\left(M_{0}\right)$ denote the exterior algebra generated by the $\left\{\omega^{i}\right\}$ over $C^{\infty}\left(M_{0}\right)$. We will refer to them as vertical forms. We can extend $d_{V}$ to a derivation

$$
d_{V}: \Omega_{V}^{p}\left(M_{0}\right) \rightarrow \Omega_{V}^{p+1}\left(M_{0}\right)
$$

by defining

$$
d_{V} \omega^{i}=-\frac{1}{2} \sum_{j, k} f_{j k}^{i} \omega^{j} \wedge \omega^{k},
$$

where the $\left\{f_{i j}{ }^{k}\right\}$ are the functions appearing in the Lie bracket of the hamiltonian vector fields associated to the constraints: $\left[X_{i}, X_{j}\right]=\sum_{k} f_{i j}{ }^{k} X_{k}$; or, equivalently, in the Poisson bracket of the constraints themselves: $\left\{\phi_{i}, \phi_{j}\right\}=\sum_{k} f_{i j}{ }^{k} \phi_{k}$.

Notice that the choice of $\left\{\omega^{i}\right\}$ corresponds to a choice of connection on the fiber bundle $M_{0} \stackrel{\pi}{\rightarrow} \tilde{M}$. Let $\mathscr{V}$ denote the subbundle of $T M_{0}$ spanned by the $\left\{X_{i}\right\}$. It can be characterized either as ker $\pi_{*}$ or as $T M_{0}^{\perp}$. A connection is then a choice of complementary subspace $\mathscr{H}$ such that $T M_{0}=\mathscr{V} \otimes \mathscr{H}$. It is clear that a choice of $\left\{\omega^{i}\right\}$ implies a choice of $\mathscr{H}$ since we can define $X \in \mathscr{H}$ if and only if $\omega^{i}(X)=0$ for all $i$. If we let $\operatorname{pr}_{V}$ denote the projection $T M_{0} \rightarrow \mathscr{V}$ it is then clear that acting on vertical forms, $d_{V}=\operatorname{pr}_{V}^{*} \circ d$, where $d$ is the usual exterior derivative on $M_{0}$.

It follows therefore that $d_{V}^{2}=0$. We call its cohomology the vertical cohomology and we denote it as $H_{V}\left(M_{0}\right)$. It turns out that it can be computed [10] in terms of the de Rham cohomology of the typical fiber in the fibration $M_{0} \stackrel{\pi}{\rightarrow} \tilde{M}$. In particular, from its definition, we already have that

$$
H_{V}^{0}\left(M_{0}\right) \cong C^{\infty}(\tilde{M})
$$

\section{BRST Cohomology: from $M$ to $\tilde{M}$}

To complete the BRST construction we must integrate the two cohomology theories into one. Corollary 2.7 suggests that we use the Koszul resolution. Notice that $\Omega_{V}\left(M_{0}\right)$ is isomorphic to $\wedge \mathbb{R}^{k} \otimes C^{\infty}\left(M_{0}\right)$, where $\mathbb{R}^{k}$ has basis $\left\{\omega^{i}\right\}$. Since the Koszul complex gives a resolution for $C^{\infty}\left(M_{0}\right)$, extending the Koszul differential as the identity on $\wedge \mathbb{R}^{k}$ yields a resolution for $\Omega_{V}\left(M_{0}\right)$. We find it convenient to think of $\mathbb{R}^{k}$ as $\mathbb{V}^{*}$, whence the resolution of $\Omega_{V}\left(M_{0}\right)$ is given by

$$
\ldots \rightarrow \bigwedge \mathbb{V}^{*} \otimes \mathbb{V} \otimes C^{\infty}(M) \stackrel{1 \otimes \delta_{K}}{\longrightarrow} \wedge \mathbb{V}^{*} \otimes C^{\infty}(M) \rightarrow 0 .
$$

This gives rise to a bigraded complex $\mathbb{K}=\bigotimes_{c, b} \mathbb{K}^{c, b}$, where

$$
\mathbb{K}^{c, b} \equiv \bigwedge^{c} \mathbb{V}^{*} \otimes \bigwedge^{b} \mathbb{V} \otimes C^{\infty}(M),
$$

under the Koszul differential $\delta_{K}: \mathbb{K}^{c, b} \rightarrow \mathbb{K}^{c, b-1}$, whose cohomology is zero for $b>0$, and for $b=0$ it is isomorphic to the vertical forms, where the vertical derivative is defined. To make contact with the usual notation, elements of $\Lambda \mathbb{V}^{*}$ (respectively, $\wedge \mathbb{V}$ ) are known as ghosts (respectively, antighosts).

The purpose of the BRST construction is to lift the vertical derivative to $\mathbb{K}$. That is, to define a differential $\delta_{1}$ on $\mathbb{K}$ which anticommutes with the Koszul differential, which induces the vertical derivative upon taking Koszul cohomology, 
and which obeys $\delta_{1}^{2}=0$. This would mean that the total differential $D=\delta_{K}+\delta_{1}$ would obey $D^{2}=0$ acting on $\mathbb{K}$ and its cohomology would be isomorphic to the vertical cohomology. Actually this is only possible in very special cases (e.g., the case of a group action). In general we will be forced to add further $\delta_{i}$ 's to $D$ to ensure $D^{2}=0$.

We find it convenient to define $\delta_{0}(-1)^{c} \delta_{K}$ on $\mathbb{K}^{b, c}$. We define $\delta_{1}$ on functions and ghosts (i.e., $\left\{\omega^{i}\right\}$ ) as the vertical derivative ${ }^{3}$

$$
\begin{aligned}
\delta_{1} f & =\sum_{i}\left(X_{i} f\right) \omega^{i} \\
& =\sum_{i}\left\{\phi_{i}, f\right\} \omega^{i}
\end{aligned}
$$

and

$$
\delta_{1} \omega^{i}=-\frac{1}{2} \sum_{j, k} f_{j k}^{i} \omega^{j} \wedge \omega^{k} .
$$

We can then extend it as a derivation to all of $\Lambda \mathbb{V}^{*} \otimes C^{\infty}(M)$. Notice that it trivially anticommutes with $\delta_{0}$ since it stabilizes $\wedge \mathbb{V}^{*} \otimes C^{\infty}(M)$, where $\delta_{0}$ acts trivially. We now define it on antighosts (i.e., $\left\{e_{i}\right\}$ ) in such a way that it commutes with $\delta_{0}$ everywhere. This does not define it uniquely but a convenient choice is

$$
\delta_{1} e_{i}=\sum_{j, k}{f_{j i}}^{k} \omega^{j} \wedge \omega^{k} .
$$

Notice that $\delta_{1}^{2} \neq 0$ in general, although it does in the case where the $f_{i j}{ }^{k}$ are constant. However since it anticommutes with $\delta_{0}$ is does induce a map in $\delta_{0}$ (i.e., Koszul) cohomology which precisely agrees with the vertical derivative $d_{V}$, which does obey $d_{V}^{2}=0$. Hence $\delta_{1}^{2}$ induces the zero map in Koszul cohomology. This is enough to deduce the existence of a derivation $\delta_{2}: \mathbb{K}^{c, b} \rightarrow \mathbb{K}^{c+2, b+1}$ such that $\delta_{1}^{2}+\left\{\delta_{0}, \delta_{2}\right\}=0$, where $\{$,$\} denotes the anticommutator. This suggests that we$ define $D_{2}=\delta_{0}+\delta_{1}+\delta_{2}$. We see that

$$
D_{2}^{2}=\delta_{0}^{2} \otimes\left\{\delta_{0}, \delta_{1}\right\} \otimes\left(\delta_{1}^{2}+\left\{\delta_{0}, \delta_{2}\right\}\right) \otimes\left\{\delta_{1}, \delta_{2}\right\} \otimes \delta_{2}^{2},
$$

where we have separated it in terms of different bidegree and arranged them in increasing $c$-degree. The first three terms are zero but, in general, the other two will not vanish. The idea behind the BRST construction is to keep defining higher $\delta_{i}: \mathbb{K}^{c, b} \rightarrow \mathbb{K}^{c+i, b+i-1}$ such that their partial sums $D_{i}=\delta_{0}+\ldots+\delta_{i}$ are nilpotent up to terms of higher and higher $c$-degree until eventually $D_{k}^{2}=0$. The proof of this statement follows easily by induction from the quasi-acyclicity of the Koszul complex. In fact, one has

Theorem 2.19. We can define a derivation $D=\sum_{i=0}^{k} \delta_{i}$ on $\mathbb{K}$, where $\delta_{i}$ are derivations of bidegree $(i, i-1)$, such that $D^{2}=0$.

\footnotetext{
${ }^{3}$ Notice that the vertical derivative is defined on $M_{0}$ and hence has no unique extension to $M$. The choice we make is the simplest and the one that, in the case of a group action, corresponds to the Lie algebra coboundary operator
} 
Defining the total complex $\mathbb{K}=\bigotimes_{n} \mathbb{K}^{n}$, where $\mathbb{K}^{n}=\bigotimes_{c-b=n} \mathbb{K}^{c, b}$, we see that $D: \mathbb{K}^{n} \rightarrow \mathbb{K}^{n+1}$. Its cohomology is therefore graded, that is, $H_{D}=\bigotimes_{n} H_{D}^{n}$. $D$ is known as the BRST operator and its cohomology is the classical BRST cohomology. The total degree is known as the ghost number. A simple spectral sequence argument yields the following theorem:

Theorem 2.20. The classical BRST cohomology is given by

$$
H_{D}^{n} \cong\left\{\begin{array}{lll}
0 & \text { for } & n<0 \\
H_{V}^{n}\left(M_{0}\right) & \text { for } & n \geq 0
\end{array}\right.
$$

In particular, $H_{D}^{0} \cong C^{\infty}(\tilde{M})$.

\section{Poisson Structure}

This, however, is not the end of the story. The reader may have noticed that so far in the construction of the BRST complex no essential use has been made of the Poisson structure of the smooth functions on $M$. Happily, it turns out that the complex $\mathbb{K}$ is a Poisson superalgebra and the BRST operator $D$ can be made into a Poisson derivation. In fact notice that $C^{\infty}(M)$ is a Poisson algebra. Moreover, if $\mathbb{V}$ is a finite dimensional vector space and $\mathbb{V}^{*}$ its dual, then the exterior algebra $\Lambda\left(\mathbb{V} \otimes \mathbb{V}^{*}\right)$ possesses a Poisson superalgebra structure. The associative multiplication is given by exterior multiplication $(\wedge)$ and the Poisson bracket is defined for $u, v \in \mathbb{V}$ and $\alpha, \beta \in \mathbb{V}^{*}$ by

$$
[\alpha, v]=\langle\alpha, v\rangle \quad[v, w]=0=[\alpha, \beta],
$$

where $\langle$,$\rangle is the dual pairing between \mathbb{V}$ and $\mathbb{V}^{*}$. We then extend it to all of $\bigwedge\left(\mathbb{V} \otimes \mathbb{V}^{*}\right)$ as an odd derivation. Therefore the classical ghosts/antighosts in BRST possess a Poisson algebra structure. In [11] it is shown that this Poisson bracket is induced from the supercommutator in the Clifford algebra $\mathrm{Cl}\left(\mathbb{V} \otimes \mathbb{V}^{*}\right)$ with respect to the non-degenerate inner product on $\mathbb{V} \oplus \mathbb{V}^{*}$ induced by the dual pairing.

Given two Poisson superalgebras $P$ and $Q$, their tensor product $P \otimes Q$ can be given the structure of a Poisson superalgebra as follows. For $a, b \in P$ and $u, v \in Q$ we define

$$
\begin{aligned}
& (a \otimes u)(b \otimes v)=(-1)^{|u||b|} a b \otimes u v, \\
& {[a \otimes u)(b \otimes v]=(-1)^{|u||b|}([a, b] \otimes u v+a b \otimes[u, v]) .}
\end{aligned}
$$

From this it follows that $\mathbb{K}=C^{\infty}(M) \otimes \wedge\left(\mathbb{V} \otimes \mathbb{V}^{*}\right)$ becomes a Poisson superalgebra.

Let $P$ be a graded Poisson superalgebra. By a Poisson derivation of degree $k$ we will mean a linear map $D: P^{n} \rightarrow P^{n+k}$ such that

$$
\begin{aligned}
D(a b) & =(D a) b+(-1)^{k|a|} a(D b), \\
D[a, b] & =[D a, b]+(-1)^{k|a|}[a, D b] .
\end{aligned}
$$

The map $a \mapsto[Q, a]$ for some $Q \in P^{k}$ automatically obeys (2.25) and (2.26). Such Poisson derivations are called inner. 
The BRST operator $D$ is clearly a derivation over the exterior product; but nothing in the way it was defined guarantees that it is a Poisson derivation and, in fact, it need not be so. However one can show that the $\delta_{i}$ 's - which are, by far, not unique - can be defined in such a way that the resulting $D$ is inner Poisson derivation. In other words, one can show the existence of the element $Q \in \mathbb{K}^{1}$ such that $D=[Q, \cdot]$. Alternatively one can construct $Q=\sum_{i \geq 0} Q_{i}$, where $Q_{i} \in \mathbb{K}^{i+1, i}$, such that $[Q, Q]=0$ and that the cohomology of the operator $[Q, \cdot]$ is isomorphic to that of $D$. This was first proven by Henneaux in [12] and later in a completely algebraic way by Stasheff in [13]. The importance of this construction lies in the following fact. Let $D=[Q, \cdot]$. Then $D^{2}=0$ and, since it is a Poisson derivation, $\operatorname{ker} D$ is a Poisson subalgebra of $\mathbb{K}$ and $\operatorname{im} D$ is a Poisson ideal of $\operatorname{ker} D$, whence its cohomology inherits naturally the Poisson algebra structure. In summary, we have the following theorem:

Theorem 2.27. The cohomology of $D$ is given by

$$
H_{D}^{n} \cong\left\{\begin{array}{lll}
0 & \text { for } & n<0 \\
H_{V}^{n}\left(M_{0}\right) & \text { for } & n \geq 0
\end{array}\right.
$$

In particular, $H_{D}^{0} \cong C^{\infty}(\tilde{M})$ as Poisson algebras.

From now on we will take $D=[Q, \cdot]$ to be the classical BRST operator and call $Q$ the classical BRST charge.

We conclude, therefore, that the classical BRST construction is completely compatible with the Poisson structure. Roughly speaking, the BRST construction feels right at home in the Poisson category.

\section{Prequantization}

Geometric quantization is an attempt to develop a mathematically consistent and invariant quantization scheme, thus trying to overcome the problems of the more traditional "canonical" quantization. Observing that the Poisson bracket was the classical analogue of the quantum commutator, Dirac reformulated the canonical quantization of a symplectic manifold $M$ as the construction of irreducible representations of the Lie algebra of real smooth functions on $M$ as self-adjoint operators in a Hilbert space with the properties that the constant function with value 1 shall be represented by the identity operator and that, if $(q, p)$ is local chart forming a canonically conjugate pair (i.e., they obey the Heisenberg algebra), then they shall act irreducibly or at least, in case one wants to include internal degrees of freedom, with finite reducibility. A celebrated theorem of Van Hove [14], however, forbids the existence of such representation unless one relaxes the finite reducibility condition. However this is demanded by the uncertainty principle and hence physically desirable. The way out is to restrict the range of functions that can be represented.

The geometric quantization program of Kostant [15] and Souriau [16] provides an invariant method of constructing such representations. The first part of the method, called prequantization, consists of dropping the irreducibility condition and constructing a representation of the Lie algebra of smooth functions as self adjoint operators in a Hilbert space, purely in terms of symplectic data. The second part of the construction, called polarization, will take care of making this 
representation irreducible and in the process restricting the class of functions which can be quantized. In this section we discuss prequentization and we see how it is intimately tied in with the notion of a Poisson module.

Let $(M, \Omega)$ be a symplectic manifold. Since $d \Omega=0$, the symplectic form defines a class in the real de Rham cohomology group $H_{\mathrm{dR}}^{2}(M ; \mathbb{R})$. We say $\Omega$ is integral (and $M$ an integral symplectic manifold) if this class lies in the image of the map

$$
H^{2}(M ; \mathbb{Z}) \rightarrow H^{2}(M ; \mathbb{R}) \cong H_{\mathrm{dR}}^{2}(M ; \mathbb{R}) .
$$

If $(M, \Omega)$ is an integral symplectic manifold then there exists at least one complex line bundle $E \rightarrow M$ with a hermitian structure, i.e., a sesquilinear map

$$
\langle,\rangle: \Gamma(E) \times \Gamma(E) \rightarrow C_{\mathbb{C}}^{\infty}(M),
$$

which is antilinear in the first factor and linear in the second; and with a connection

$$
\nabla: \Gamma(E) \rightarrow \Omega^{1}(M) \otimes \Gamma(E),
$$

such that

(PQ1) $\langle$,$\rangle is parallel with respect to \nabla$; that is, for all $\sigma, \tau \in \Gamma(E)$,

$$
d\langle\sigma, \tau\rangle=\langle\sigma, \nabla \tau\rangle+\langle\nabla \sigma, \tau\rangle
$$

(PQ2) the symplectic form and the curvature 2-form of the connection are related by

$$
\operatorname{curv}(\nabla)=-2 \pi \sqrt{-1} \Omega \text {. }
$$

The triple $(E, \nabla,\langle\rangle$,$) satisfying the above properties will be called pre-$ quantum data for the integral symplectic manifold $(M, \Omega)$.

Let $d \mu_{L}$ denote the Liouville measure on $M$. This is the measure induced by the volume form proportional to $\underbrace{\Omega \wedge \ldots \wedge \Omega}_{n}$ for $M$ a $2 n$-dimensional manifold. This allows us to define an inner product on $\Gamma(E)$ by integrating the pointwise inner product with respect to this measure:

$$
(\sigma, \tau) \equiv \int_{M}\langle\sigma, \tau\rangle d \mu_{L}
$$

Let $\Gamma_{L^{2}}(E)$ denote the Hilbert space completion of the subspace of $\Gamma(E)$ consisting of sections $\sigma$ such that $\|\sigma\|^{2} \equiv(\sigma, \sigma)<\infty$. This will become the prequantum Hilbert space. The prequantization map assigning to a smooth function $f$ an operator $O(f)$ in $\Gamma_{L^{2}}(E)$ is the following

$$
f \mapsto O(f) \equiv \nabla_{X_{f}}+2 \pi \sqrt{-1} f,
$$

where $X_{f}$ is the Hamiltonian vector field associated to $f$, that is, $i\left(X_{f}\right) \Omega+d f=0$. The prequantization map obeys the following

$$
\begin{gathered}
O(f) O(g) \sigma-O(g) O(f) \sigma=O(\{f, g\}) \sigma, \\
O(f)(g \sigma)=\{f, g\} \sigma+g O(f) \sigma,
\end{gathered}
$$

for all $\sigma \in \Gamma(E)$ and $f, g \in C^{\infty}(M)$. Moreover each $O(f)$ is a skew-symmetric operator. That is, if $\sigma, \tau \in \Gamma_{L^{2}}(E)$ are in the domain of $O(f)$ than

$$
(O(f) \sigma, \tau)+(\sigma, O(f) \tau)=0 .
$$


If, in addition, $X_{f}$ is a complete vector field, $O(f)$ has a skew-self-adjoint extension and generates, by Stone's theorem, a one parameter family of unitary operators in $\Gamma_{L^{2}}(E)$.

The prequantization map has the property that the only operator of the form $O(f)$ for some $f \in C^{\infty}(M)$ which commutes with all the other $O(g)$ 's are the scalars, corresponding to $O(c)$ for $c$ a constant function on $M$. Still this representation is highly reducible: roughly speaking it consists of integrable functions of both the momenta and the coordinates. Thus we need to cut down the size of $\Gamma_{L^{2}}(E)$. This process, known as polarization, will be the topic of the second part of this paper [3]. In the rest of this section we first show how prequentization is tied to the notion of a Poisson module and how this suggests how to define the prequentum BRST operator.

The notion of a Poisson module is fairly simple. A Poisson superalgebra consists of a vector space in which one has defined two algebraic structures subject to a compatibility condition. On the one hand a Poisson superalgebra is a supercommutative associative algebra but also a Lie superalgebra. The compatibility condition consists in demanding that the adjoint action of the Lie superalgebra on itself be a derivation over the associative structure. We can analogously define a Poisson module over a Poisson superalgebra $P$ as a $\mathbb{Z}_{2^{-}}$ graded vector space which is both a module over the associative part of $P$ and separately a module over the Lie part of $P$. We then impose a compatibility condition deduced from the compatibility condition between the two algebraic structure in $P$. More formally we say that a $\mathbb{Z}_{2}$-graded vector space $M$ is a Poisson module over a Poisson superalgebra $P$, if there exist two bilinear operations preserving the grading

and

$$
\begin{aligned}
P \times M & \rightarrow M \\
(a, m) & \mapsto a \cdot m
\end{aligned}
$$

$$
\begin{aligned}
P \times M & \rightarrow M \\
(a, m) & \mapsto a \times m,
\end{aligned}
$$

obeying the following properties

(M1) - makes $M$ a module over the associative structure of $P$ :

$$
a \cdot(b \cdot m)=(a b) \cdot m
$$

(M2) $\times$ makes $M$ into a module over the Lie superalgebra structure of $P$ :

$$
a \times(b \times m)-(-1)^{|a||b|} b \times(a \times m)=[a, b] \times m ;
$$

(M3) For all $a, b \in P$ and $m \in M$,

$$
a \times(b \cdot m)=[a, b] \cdot m+(-1)^{|a||b|} b \cdot(a \times m) .
$$

In particular a Poisson algebra becomes a Poisson module over itself after identifying $a \cdot b$ with $a b$ and $a \times b$ with $[a, b]$. Notice that Eqs. (3.6) and (3.7) imply that $\Gamma(E)$ becomes a Poisson module over $C^{\infty}(M)$ and in particular that the prequantization may defined by (3.5) is nothing but the map $a \mapsto a \times$.

This observation immediately suggests how to prequentize the classical BRST operator. Just like the tensor product of two Poisson superalgebras can be made into a Poisson superalgebra, if $M$ and $N$ are Poisson modules over $P$ and $Q$, 
respectively, their tensor product $M \otimes N$ becomes a $P \otimes Q$-module under the following operations:

$$
\begin{aligned}
(a \otimes u) \cdot(m \otimes n) & =(-1)^{|u| \mid m}(a \cdot m) \otimes(u \cdot n), \\
(a \otimes u) \times(m \otimes n) & =(-1)^{|u||m|}(a \times m \otimes u \cdot n+a \cdot m \otimes u \times n),
\end{aligned}
$$

for all $a \in P, u \in Q, m \in M$, and $n \in N$. Since $\Lambda\left(\mathbb{V} \otimes \mathbb{V}^{*}\right)$ is a Poisson module over itself and $\Gamma(E)$ is a Poisson module over $C^{\infty}(M)$, their tensor product $\mathbb{K}(E) \equiv \Gamma(E) \otimes \bigwedge\left(\mathbb{V} \otimes \mathbb{V}^{*}\right)$ becomes a Poisson module over $\mathbb{K}$. Not let $Q \in \mathbb{K}^{1}$ be the classical BRST charge. Then the prequantum BRST operator is the operator $\mathbb{D} \equiv Q \times \cdot$ acting on $\mathbb{K}(E)$. Since $[Q, Q]=0, \mathbb{D}^{2}=0$ and we may define its cohomology $H_{\mathbb{D}}$, as the prequantum BRST cohomology, which we will investigate in the next section. Notice that it follows from (M1)-(M3) that $H_{\mathbb{D}}$, inherits the structure of a graded Poisson module over $H_{D}$. In particular, $H_{\mathbb{D}}^{0}$ is a Poisson module over $H_{D}^{0}$. In the next section we will see that it indeed the module of sections of a prequantum line bundle over the reduced symplectic manifold.

\section{Prequantum BRST Cohomology}

In this section we compute the cohomology $H_{\mathbb{D}}$ of the prequantum BRST operator and show that $H_{\mathrm{D}}^{0}$ is isomorphic as a Poisson module to the space of smooth sections of a prequantum line bundle over the reduced symplectic manifold $\tilde{M}$. We also contrast this result with the similar results of Guillemin and Sternberg [1].

Recall that $\mathbb{K}(E)$ is bigraded by

$$
\mathbb{K}(E)=\bigotimes_{b, c} \mathbb{K}^{c, b}(E), \quad \mathbb{K}^{c, b}(E) \equiv \bigwedge^{c} \mathbb{V}^{*} \otimes \bigwedge^{b} \mathbb{V} \otimes \Gamma(E)
$$

Relative to this bigrading $\mathbb{D}$ breaks up as $\mathbb{D}=\sum_{i \geq 0} \nabla_{i}$, where $\nabla_{i}: \mathbb{K}^{c, b}(E) \rightarrow$ $\mathbb{K}^{c+i, b+i-1}(E)$. The $\nabla_{i}$ can be recovered from the $Q_{i}$ in $Q=\sum_{i \geq 0} Q_{i}$ by picking the contributions of the right bidegree. From the expressions for $\delta_{0}$ and $\delta_{1}$ given by (2.3), (2.15), (2.16), and (2.17) we can work out $Q_{0}$ and $Q_{1}$ :

$$
\begin{aligned}
& Q_{0}=\sum_{i} \omega^{i} \phi_{i}, \\
& Q_{1}=-\frac{1}{2} \sum_{i, j, k} f_{i j}{ }^{k} \omega^{i} \wedge \omega^{j} e_{k} .
\end{aligned}
$$

This, in turns, allows us to write $\nabla_{0}$ and $\nabla_{1}$ on generators. First of all $\nabla_{0}$ is a trivial extension of the Koszul differential $\delta_{K}$. In fact, $\nabla_{0}=(-1)^{c} \delta_{K} \otimes \mathbf{1}$ on $\mathbb{K}^{c, b}(E)$. In other words, $\nabla_{0}$ is zero on sections and on ghosts and it is up to a sign the Koszul differential on antighosts. Similarly we find that on generators 
$\nabla_{1}$ acts as follows:

and

$$
\begin{aligned}
\nabla_{1} \sigma & =\sum_{i}\left(\phi_{i} \times \sigma\right) \omega^{i} \\
\nabla_{1} \omega^{i} & =-\frac{1}{2} \sum_{j, k} f_{j k}^{i} \omega^{j} \wedge \omega^{k}
\end{aligned}
$$

$$
\nabla_{1} e_{i}=\sum_{j, k} f_{j i}{ }^{k} \omega^{j} \wedge \omega_{k}
$$

In particular, splitting $\mathbb{D}^{2}=0$ into different bidegrees, we find that

$$
\begin{aligned}
\nabla_{0}^{2} & =0, \\
{\left[\nabla_{0}, \nabla_{1}\right] } & =0, \\
\nabla_{1}^{2}+\left[\nabla_{2}, \nabla_{0}\right] & =0 .
\end{aligned}
$$

We now define $F^{p} \mathbb{K}(E) \equiv \bigotimes_{b} \bigotimes_{c \geq p} \mathbb{K}^{c, b}(E)$. Then $\mathbb{K}(E)=F^{0} \mathbb{K}(E) \supseteq F^{1} \mathbb{K}(E) \supseteq$ .. and $\mathbb{D F}^{p} \mathbb{K}(E) \subseteq F^{p} \mathbb{K}(E)$. In other words, $(F \mathbb{K}(E), \mathbb{D})$ is a filtered complex. Since the filtration is bounded there exists a spectral sequence converging finite to $H_{\mathbb{D}}$ whose $E_{1}$ term is the cohomology of the associated graded complex. The differential is the induced one from $\mathbb{D}$ and it is clearly seen to be $\nabla_{0}$, since $\nabla_{i}$ for $i>0$ all have positive filtration degrees. Therefore $\underset{b}{\bigotimes} E_{1}^{c, b}$ is the cohomology of
the generalized Koszul complex

$$
\cdots \rightarrow \bigwedge^{c} \mathbb{V}^{*} \otimes \mathbb{V} \otimes \Gamma(E) \stackrel{1 \otimes \delta_{K}}{\longrightarrow} \bigwedge^{c} \mathbb{V}^{*} \otimes \Gamma(E) \rightarrow 0
$$

Observing that $\Gamma(E)$, being the module of smooth sections of a vector bundle, is a finitely generated projective module over $C^{\infty}(M)$ we can use the results of Appendix A to conclude that

$$
E_{1}^{c, b} \cong\left\{\begin{array}{lll}
\bigwedge^{c} \mathbb{V}^{*} \otimes \Gamma(E) / J \Gamma(E) & \text { for } & b=0 \\
0 & \text { for } & b>0
\end{array}\right.
$$

We now turn to the computation of $\Gamma(E) / J \Gamma(E)$. Let $i: M_{0} \rightarrow M$ denote the natural inclusion. Then if we let $i^{-1} E \rightarrow M_{0}$ denote the pull-bach via $i$ of the prequantum line bundle $E \stackrel{\pi}{\rightarrow} M$, it will follow from the following theorem that $\Gamma(E) / J \Gamma(E)$ is isomorphic to $\Gamma\left(i^{-1} E\right)$. But first we need some remarks of a more general nature.

Let $\psi: M^{\prime} \rightarrow M$ be a smooth map between differentiable manifolds. It induces a ring homorphism

$$
\psi^{*}: C^{\infty}(M) \rightarrow C^{\infty}\left(M^{\prime}\right)
$$

defined by $\psi^{*} f=f \circ \psi$ for $f \in C^{\infty}(M)$. This makes any $C^{\infty}\left(M^{\prime}\right)$-module (in particular $C^{\infty}\left(M^{\prime}\right)$ itself) into a $C^{\infty}(M)$-module, by restriction of scalars: multiplication by $C^{\infty}(M)$ is effected by precomposing multiplication by $C^{\infty}\left(M^{\prime}\right)$ with $\psi^{*}$. 
Now let $E^{\prime} \stackrel{\pi^{\prime}}{\rightarrow} M^{\prime}$ and $E \stackrel{\pi}{\rightarrow} M$ be vector bundles of the same rank with the property that there is a bundle map given by the following commutative diagram

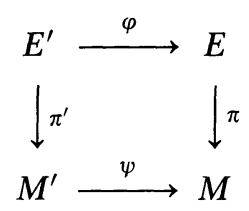

(i.e., $\varphi$ is smooth fiber-preserving) with the property that $\varphi$ restricts to a linear isomorphism on the fibers. Then we may form the following $C^{\infty}(M)$-module

$$
C^{\infty}\left(M^{\prime}\right) \otimes_{C^{\infty}(M)} \Gamma(E),
$$

which can be made into a $C^{\infty}\left(M^{\prime}\right)$-module by extension of scalars: left multiplication by $C^{\infty}\left(M^{\prime}\right)$. Define a map $\varphi^{\#}: \Gamma(E) \rightarrow \Gamma\left(E^{\prime}\right)$ by

$$
\left(\varphi^{\#} \sigma\right)\left(m^{\prime}\right)=\left(\varphi_{m^{\prime}}\right)^{-1}\left[\sigma\left(\psi\left(m^{\prime}\right)\right)\right],
$$

for all $m^{\prime} \in M^{\prime}$ and $\sigma \in \Gamma(E)$. Then the following can be easily proven [17]:

Theorem 4.16. With the above notation, there exists an isomorphism of $C^{\infty}\left(M^{\prime}\right)$ modules

$$
C^{\infty}\left(M^{\prime}\right) \otimes \Gamma(E) \rightarrow \Gamma\left(E^{\prime}\right) ;
$$

defined by $f \otimes \sigma \mapsto f \cdot \varphi^{\#} \sigma$ and where the tensor product is over $C^{\infty}(M)$.

In our case we have the following commutative bundle diagram

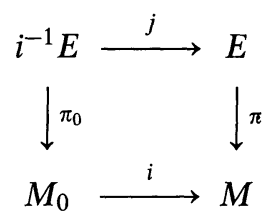

By Theorem 4.16, we have that

$$
\Gamma\left(i^{-1} E\right) \cong C^{\infty}\left(M_{0}\right) \otimes_{C^{\infty}(M)} \Gamma(E) .
$$

But $C^{\infty}\left(M_{0}\right) \cong C^{\infty}(M) / J$, whence

$$
\begin{aligned}
\Gamma\left(i^{-1} E\right) & \cong C^{\infty}(M) / J \otimes_{C^{\infty}(M)} \Gamma(E) \\
& \cong \Gamma(E) / J \Gamma(E),
\end{aligned}
$$

where the last isomorphism is standard. In other words, letting $E_{0} \equiv i^{-1} E$, the $E_{1}$ term of the spectral sequence is given by

$$
E_{1}^{c, b} \cong \begin{cases}\bigwedge^{c} \mathbb{V}^{*} \otimes \Gamma\left(E_{0}\right) & \text { for } b=0 \\ \mathbf{0} & \text { for } b>0,\end{cases}
$$

and that, as a $C^{\infty}\left(M_{0}\right)$ module, $E_{1}$ is isomorphic to the module $\Omega_{V}\left(E_{0}\right)$ of vertical forms with coefficients in $E_{0}$, which is defined as

$$
\Omega_{V}\left(E_{0}\right) \equiv \Omega_{V}\left(M_{0}\right) \otimes_{C^{\infty}\left(M_{0}\right)} \Gamma\left(E_{0}\right)
$$


Tracking down the definitions we see that the $E_{2}$ term in the spectral sequence is precisely the cohomology of $E_{1}$ with respect to the differential $\left(\nabla_{1}\right) *$ induced by $\nabla_{1}$. (Notice that $E_{1}$ is the cohomology of $\nabla_{0}$ and that by (4.8) and (4.9), $\nabla_{1}$ induces a map on $E_{1}$ which is square-zero.) Under the isomorphism $E_{1} \cong \Omega_{V}\left(E_{0}\right)$ we see that $\left(\nabla_{1}\right) *$ goes over to the operator $\nabla_{V}$ defined by

$$
\nabla_{V} \sigma=\sum_{i}\left(\phi_{i} \times \sigma\right) \omega^{i}
$$

and

$$
\nabla_{V} \omega^{i}=-\frac{1}{2} \sum_{j, k}{f_{j k}}^{i} \omega^{j} \wedge \omega^{k},
$$

for all $\sigma \in \Gamma\left(E_{0}\right)$. This is then extended to all of $\Omega_{V}\left(E_{0}\right)$ as a derivation. This operator is an extension of the vertical derivative $d_{V}$ and, just as for $d_{V}$, it is easy to verify that $\nabla_{V}^{2}=0$. We denote its cohomology by $H_{V}\left(E_{0}\right)$. Moreover notice that for all $E_{0}$-valued vertical forms $\theta$

$$
\nabla_{V}(f \theta)=d_{V} f \wedge \theta+f \nabla_{V} \theta,
$$

whence, in particular, $H_{V}^{0}\left(E_{0}\right)$ becomes a $C^{\infty}(\tilde{M})$-module (under pointwise multiplication) after the identification of $C^{\infty}(\tilde{M})$ with $H_{V}^{0}\left(M_{0}\right)$. To see this notice that if $d_{V} f=0$ and $\nabla_{V} \sigma=0$, for some $\sigma \in \Gamma\left(E_{0}\right), \nabla_{V}(f \sigma)=0$. Moreover, it is easy to verify that this module is finitely generated and projective. Hence, by general arguments [17], it is the module of sections of some vector bundle over $\tilde{M}$. Moreover Guillemin and Sternberg show in (3.2)-(3.4) of [1] that the bundle $\tilde{E}$ is in fact a prequantum line bundle over $\tilde{M}$. It is, in fact, the bundle $\tilde{E} \rightarrow \tilde{M}$ whose pullback to $M_{0}$ is isomorphic (as a complex line bundle with connection) to the bundle $E_{0} \rightarrow M_{0}$.

Therefore we have proven that $E_{2} \cong H_{V}\left(E_{0}\right)$. Furthermore since $E_{1}^{c, b}=0$ for $b>0$, the spectral sequence collapses at the $E_{2}$ term, whence

Theorem 4.26. The cohomology of $\mathbb{D}$ is given by

$$
H_{\mathbb{D}}^{n} \cong\left\{\begin{array}{lll}
0 & \text { for } & n<0 \\
H_{V}^{n}\left(E_{0}\right) & \text { for } & n \geq 0 .
\end{array}\right.
$$

In particular, $H_{\mathbb{D}}^{0} \cong \Gamma(\tilde{E})$, where the isomorphism is one of $C^{\infty}(\tilde{M})$ modules.

As remarked earlier $H_{\mathbb{D}}^{0}$ inherits the structure of a Poisson module over $H_{D}^{0}$ which, by Theorem 2.27 , is isomorphic as a Poisson algebra to $C^{\infty}(\tilde{M})$. We still have to show that the isomorphism $\Gamma(\tilde{E}) \cong \mathscr{H}_{\mathrm{D}}^{0}$ is one of Poisson modules. In other words, we must check that under the Poisson algebra isomorphism $H_{D}^{0} \cong C^{\infty}(\tilde{M})$ the actions correspond. This requires a better understanding of the isomorphisms $H_{D}^{0} \cong C^{\infty}(\tilde{M})$ and $H_{\mathrm{D}}^{0} \cong \Gamma(\tilde{E})$. Space constraints force us to be sketchy, and we refer the reader to [8] for a more thorough discussion.

A function $f \in C^{\infty}(\tilde{M})$ can be represented by a function $f_{0} \in C^{\infty}(M)$ whose Poisson brackets with the constraints vanish on $M_{0}$; that is, they lie in the ideal $J$ generated by the constraints. Such functions celarly comprise $N(J) / J$, where $N(J)$ is the normalizer of $J$ in $C^{\infty}(M)$. In other words, $f_{0}$ satisfied $\delta_{0} f_{0}=0$ and $\delta_{1} f_{0}+\delta_{0} f_{1}=0$ for some $f_{1} \in \mathbb{K}^{1,1}$. It is easy to show (by induction) that we can complete $f_{0}$ and $f_{1}$ to an element $F=f_{0} \oplus f_{1} \oplus \ldots \in \mathbb{K}^{0}$ with $f_{i} \in \mathbb{K}^{i, i}$ such that $D F=0$. Hence this assigns to an element of $C^{\infty}(\tilde{M})$ a cocycle in $\mathbb{K}^{0}$. It is also easy to show that this map is well defined and that in fact it descends to an 
injective map in cohomology. Conversely, given a cohomology class in $H_{D}^{0}$ with representative cocycle $g_{0} \oplus g_{1} \oplus \ldots, g_{0}$ is a function on $C^{\infty}(M)$ whose Poisson brackets with the constraints vanish on $M_{0}$.

We can now outline the proof of the Poisson algebra isomorphism between $H_{D}^{0}$ and $C^{\infty}(\tilde{M})$. First notice that as Poisson algebras $C^{\infty}(\tilde{M})$ is isomorphic to $N(J) / J$. Now let $f$ and $g$ be representatives in $N(J)$ of classes in $N(J) / J$ and let them be mapped into $D$-cocycles $F=f \oplus \ldots$ and $G=g \oplus \ldots$ respectively. Then their product is a $D$-cocycle of the form $F G=f g \oplus \ldots$ - whence it corresponds to $f g$ - and their Poisson bracket is a $D$-cocycle $\{F, G\}=\{f, g\} \oplus \ldots$ - whence it corresponds to $\{f, g\}$. Therefore the Poisson structures correspond.

Similarly we can prove that $H_{\mathbb{D}}^{0}$ is isomorphic to $\Gamma(\tilde{E})$ as Poisson modules over $C^{\infty}(\tilde{M})$ under the isomorphism $H_{D}^{0} \cong C^{\infty}(\tilde{M})$ just described. Given a section $\sigma \in \Gamma(\tilde{E})$ it can be represented by a section $\sigma_{0} \in \Gamma(E)$ obeying $\nabla_{1} \sigma_{0}+\nabla_{0} \sigma_{1}=0$ for some $\sigma_{1} \in \mathbb{K}^{1,1}(E)$. Just as for the case of functions it can be completed into a $\mathbb{D}$-cocycle $\Sigma=\sigma_{0} \oplus \sigma_{1} \oplus \ldots$ and again the correspondence descends to cohomology. Conversely, given a cohomology class in $H_{\mathbb{D}}^{0}$ represented by the cocycle $\sigma_{0} \oplus \sigma_{1} \oplus \ldots, \sigma_{0}$ satisfies $\nabla_{1} \sigma_{0}+\nabla_{0} \sigma_{1}=0$; and it therefore defines a section in $\Gamma(\tilde{E})$. Now let $f \in N(J)$ represent a function in $C^{\infty}(\tilde{M})$ and be mapped into the $D$-cocycle $F \in \mathbb{K}^{0}$. Then the $\mathbb{D}$-cocycles $F \Sigma=f \sigma_{0} \oplus \ldots$ and $F \times \Sigma=f \times \sigma_{0} \oplus \ldots$ correspond to the sections $f \sigma_{0}$ and $f \times \sigma_{0}$. This proves the isomorphism of $H_{\mathbb{D}}^{0}$ and $\Gamma(\tilde{E})$ as Poisson modules over $C^{\infty}(\tilde{M})$.

There is one final step we must take in order to conclude that all prequantum data gets induced via BRST and that is to make sure that the point-wise inner product gets induced as well. In order to induce a pointwise inner product on $H_{\mathbb{D}}^{0}$ it will be first of all necessary to define a pointwise inner product on $\mathbb{K}(E)$. To motivate this construction let us first understand in Poisson terms the invariance of the pointwise inner product of two invariant sections. This invariance follows from the following fact. Since $\langle$,$\rangle is \mathbb{R}$-linear in both slots it induces a map

$$
\langle,\rangle: \Gamma(E) \otimes \Gamma(E) \rightarrow C_{\mathbb{C}}^{\infty}(M),
$$

which is a $C^{\infty}(M)$-module homomorphism (a homomorphism of Poisson modules over $\left.C^{\infty}(M)\right)$. That is, if $\sigma, \tau \in \Gamma(E)$ and $f \in C^{\infty}(M)$ then

$$
[f,\langle\sigma, \tau\rangle]=\langle f \times \sigma, \tau\rangle+\langle\sigma, f \times \tau\rangle .
$$

We would now like to extend $\langle$,$\rangle to a \mathbb{K}$-module homomorphism

$$
\left\langle\langle,\rangle: \mathbb{K}(E) \otimes \mathbb{K}(E) \rightarrow \mathbb{K}_{\mathbb{C}} .\right.
$$

This boils down, essentially, to defining a linear map

$$
\langle,\rangle: \Lambda\left(\mathbb{V} \otimes \mathbb{V}^{*}\right) \otimes \Lambda\left(\mathbb{V} \otimes \mathbb{V}^{*}\right) \rightarrow \Lambda\left(\mathbb{V} \otimes \mathbb{V}^{*}\right),
$$

satisfying, for all $\phi, \omega, \theta \in \Lambda\left(\mathbb{V} \otimes \mathbb{V}^{*}\right)$, the following relations

and

$$
\langle\phi \wedge \omega, \theta\rangle=\phi \wedge\langle\omega, \theta\rangle=(-1)^{|\phi||\omega|}\langle\omega, \phi \wedge \theta\rangle,
$$

$$
[\phi,\langle\omega, \theta\rangle]=\langle[\phi, \omega], \theta\rangle+(-1)^{|\phi||\omega|}\langle\omega,[\phi, \theta]\rangle .
$$

There is one obvious candidate:

$$
\langle\omega, \theta\rangle=\omega \wedge \theta .
$$


With this choice we can construct a sesquilinear map

$$
\langle,\rangle: \mathbb{K}(E) \times \mathbb{K}(E) \rightarrow \mathbb{K}_{\mathbb{C}},
$$

which is invariant under the action of $\mathbb{K}$. It is then clear that, if $Z(E)$ and $B(E)$ stand for the $\mathbb{D}$ cocycles and coboundaries respectively and $Z$ and $B$ stand for the $D$ cocycles and coboundaries respectively, the mapping $\langle$,$\rangle obeys$

$$
\begin{aligned}
& Z(E) \times Z(E) \rightarrow Z, \\
& Z(E) \times B(E) \rightarrow B, \\
& B(E) \times Z(E) \rightarrow Z ;
\end{aligned}
$$

from where it follows that it induces a well defined map in cohomology. In particular, since it is graded, it induces a map

$$
\widetilde{\langle,\rangle}: H_{\mathbb{D}}^{0} \times H_{\mathbb{D}}^{0} \rightarrow H_{D}^{0} \otimes \mathbb{C},
$$

which, under the relevant identifications, becomes a pointwise inner product

$$
\widetilde{\langle,\rangle}: \Gamma(\tilde{E}) \times \Gamma(\tilde{E}) \rightarrow C_{\tilde{C}}^{\infty}(\tilde{M}) .
$$

It is interesting to notice, however, that there is no inner product on $\mathbb{K}(E)$ which induces, by evaluating it on $\mathbb{D}$ cocycles, the prequantum inner product on $\tilde{M}$. The reason is the following. The inner product consists of integrating the pointville inner product with respect to the Liouville measure. It is impossible that one can evaluate the inner product of sections of the prequantum bundle on $\tilde{M}$ by merely picking representative sections on $M$ and evaluating the inner product there. The reason being that functions on $\tilde{M}$ are represented by functions on $M$ whose restriction to $M_{0}$ are constant on the leaves of the null foliation. But $M_{0}$ has Liouville measure zero in $M$ and hence two functions which agree on $M_{0}$ but which disagree at will away from $M_{0}$ have different integrals. Therefore the inner product would not be independent of the representatives. By tensoring the sections of the prequantum line bundle with half-forms (see [18]) the prequantum BRST cohomology of this new complex yields objects whose pointwise inner product can be integrated on $\tilde{M}$ but, again, the integral does not lift to $M$.

\section{Conclusions}

In this paper we have proven that prequantization and reduction commute. In other words, we have shown how the following diagram related to (1.1) is commutative:

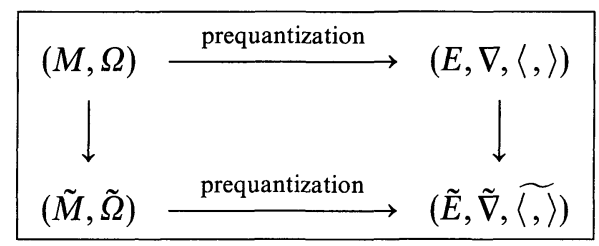

provided that by $(\tilde{E}, \tilde{\nabla})$ we take the prequantum line bundle whose pullback to $M_{0}$ agrees (as a line bundle with connection) with the pullback to $M_{0}$ of the prequantum line bundle $(E, \nabla)$ on $M$. This is a first step towards the more difficult question on the commutativity of (1.1). 
The proof of commutativity is also extremely natural once the relevant structures are phrased in Poisson terms. In fact, the coherence between the BRST construction and the Poisson structures suggests that we can phrase this construction in purely algebraic terms in the category of "constrained" Poisson algebras $[13,19]$. That is, given a Poisson algebra $P$ with a distinguished set of elements $\Phi=\left\{\phi_{i}\right\}$ such that the ideal $J$ they generate is a Lie subalgebra of $P$ and such that the sequence $\Phi$ is regular we can construct a BRST cohomology theory which reduces $P$ to another Poisson algebra. This is precisely the point of view taken by Stasheff $[13,19]$. We can extend this further by homologically reducing Poisson modules of $P$. In the same way we show that a BRST theory exists which reduces the Poisson module into a Poisson module of the homological reduction of $P$. Moreover properties such as finitely generated and projective are preserved under reduction. Also the algebraic analog of point-wise inner product can be also induced.

The power of the algebraic approach lies in the fact that all the objects in the geometric quantization program can be defined purely algebraically without the assumption that the Poisson algebra $P$ or its homological reduction are the algebras of functions on a symplectic manifold [20]. This is the case, for example, in Yang-Mills, where the reduced phase space has singularities [21]. We think that this particular formulation of BRST quantization can play a fundamental rôle in the quantization of such physically relevant systems.

Finally there are several obvious extensions to these constructions: to the case of supermanifolds in the sense of Kostant, to arbitrary symplectic Banach manifolds, and, more importantly, to the case of nonregular constraints [22]. The extensions to supermanifolds and to the non-regular case should be completely straightforward; but the extension to infinite dimensional manifolds may pose problems having to do with the convergence of the spectral sequence used in Sect. 4.

\section{Appendix A. Generalized Koszul Complex}

Let $R$ be a ring and $E$ and $R$-module. We can then define a complex $K(\Phi ; E)$ associated to any sequence $\left(\phi_{1}, \ldots, \phi_{k}\right)$ by just tensoring the Koszul complex $K(\Phi)$ with $E$, that is, $K^{p}(\Phi ; E)=K^{p}(\Phi) \otimes_{R} E$ and extending $\delta_{K}$ to $\delta_{K} \otimes 1$. Let $H(K(\Phi) ; E)$ denote the cohomology of this complex. It is naturally an $R$-module. It is easy to show that if $E$ and $F$ are $R$-modules, then there is an $R$-module isomorphism

$$
H(K(\Phi) ; E \otimes F) \cong H(K(\Phi) ; E) \otimes H(K(\Phi) ; F) .
$$

Hence, if $F=\bigotimes_{\alpha} R$ is a free $R$-module then

$$
H(K(\Phi) ; F) \cong \bigotimes_{\alpha} H(K(\Phi))
$$

In particular if $\Phi$ is a regular sequence then the generalized Koszul complex with coefficients in a free $R$-module is quasi-acyclic. Now let $P$ be a projective module, i.e., $P$ is a summand of a free module. Then let $N$ be an $R$-module such that $P \otimes N=F, F$ a free $R$-module. Then

$$
H(K(\Phi) ; F) \cong H(K(\Phi) ; P) \otimes H(K(\Phi) ; N),
$$


which, along with the quasi-acyclicity of $H(K(\Phi) ; F)$, implies the quasi-acyclicity of $H(K(\Phi) ; P)$. How about $H^{0}(K(\Phi) ; P)$ ? By definition

$$
H^{0}(K(\Phi) ; P) \cong R / J \bigotimes_{R} P \cong P / J P
$$

Therefore we have the following algebraic result.

Theorem A.5. If $\Phi=\left(\phi_{1}, \ldots, \phi_{k}\right)$ is a regular sequence in $R$, and $P$ is a projective $R$-module, then the homology of the Koszul complex with coefficients in $P$ is given by

$$
H^{p}(K(\Phi) ; P) \cong\left\{\begin{array}{lll}
0 & \text { for } & p>0 \\
P / J P & \text { for } & p=0
\end{array}\right.
$$

where $J$ is the ideal generated by the $\phi_{i}$.

The relevance of this construction is that the smooth sections of any vector bundle over $M$ have the structure of a (finitely generated) projective $C^{\infty}(M)$ module. More precisely, let $E \stackrel{\pi}{\rightarrow} M$ be a complex vector bundle of rank $r$ over $M$ and let $\Gamma(E)$ denote the space of smooth sections. It is clear that $\Gamma(E)$ is a module over $C^{\infty}(M)$, where multiplication is defined pointwise using the linear structure on each fiber. It is straightforward to prove that $\Gamma(E)$ is a free rank $r C^{\infty}(M)$-module if and only if $E$ is a trivial bundle. It can be shown [17] that $M$ has a finite cover trivializing $E$. Since on each set of the cover, $E$ is trivial we see that $\Gamma(E)$ is finitely generated: just take as a set of generators the local sections on each cover multiplied by the appropriate elements of a partition of unity subordinate to the cover.

It can also be shown [17] that given a vector bundle $E \stackrel{\pi}{\rightarrow} M$ there exists another vector bundle $F \stackrel{\varrho}{\rightarrow} M$ such that their Whitney sume $E \otimes F$ is trivial. Therfore $\Gamma(E \otimes F) \cong \Gamma(E) \otimes \Gamma(F)$ is a free $C^{\infty}(M)$ module and we that $\Gamma(E)$ is a direct summand of a free module. In summary, $\Gamma(E)$ is a finitely generated projective $C^{\infty}(M)$-module.

Acknowledgements. We are very grateful to Marc Henneaux and Johannes Huebschmann for their valuable comments on an earlier draft of this manuscript. We also appreciate conversations with E. Barrios, Francisco Figueirido, Krzysztof Galicki, Blaine Lawson, and Eduardo Ramos.

\section{References}

1. Guillemin, V., Sternberg, S.: Invent. Math. 67, 515 (1982)

2. Duval, C., Elhadad, J., Tuynman, G.M.: CPT Marseille Preprint CPT-89/P.2248

3. Figueroa-O'Farrill, J.M., Henneaux, M., Kimura, T.: In preparation

4. Huebschmann, J., Poisson cohomology and quantization, Heidelberg Preprint 1989

5. Guillemin, V., Sternberg, S.: Symplectic techniques in physics. Cambridge: Cambridge University Press, 1984

6. Marsden, J., Weinstein, A.: Rep. Math. Phys. 5, 121 (1974)

7. Batalin, I.A., Vilkovisky, G.A.: Phys. Lett. B 69, 309 (1977); Henneaux, M.: Phys. Rep. 126, 1 (1985); McMullan, D.: J. Math. Phys. 28, 428 (1987); Browning, A.D., McMullan, D.: J. Math. Phys. 28, 438 (1987); Kostant, B., Sternberg, S.: Ann. Phys. 176, 49 (1987); Stasheff, J.: Bull. Am. Math. Soc. 19, 287 (1988); Dubois-Violette, M.: Ann. Inst. Fourier 37, (4),45 (1987); Henneaux, M., Teitelboim, C.: Commun. Math. Phys. 115, 213 (1988)

8. Figueroa-O'Farrill, J.M., Kimura, T.: Stony Brokk Preprint ITP-SB-88-81 (rev.)

9. Lang, S.: Algebra. Reading, MA: Addison-Wesley 1984 
10. Figueroa-O'Farrill, J.M.: The topological characterization of classical BRST cohomology. Commun. Math. Phys. 127, 181-186 (1990)

11. Kostant, B., Sternberg, S.: Ann. Phys. 176, 49 (1987)

12. Henneaux, M.: Phys. Rep. 126, 1 (1985)

13. Stasheff, J.: Bull. Am. Math. Soc. 19, 287 (1988)

14. Van Hove, L.: Acad. Roy. Belgique Bull. Sci. 37 (5), 610 (1951); Abraham, R., Marsden, J.: Foundations of mechanics. New York, NY: Benjamin 1978

15. Kostant, B.: Quantization and unitary representations. I. Prequantization. In: Lecture Notes in Math. Vol. 170, pp. 87:208. Berlin, Heidelberg, New York: Springer 1970

16. Souriau, J.-M.: Structure des systèmes dynamiques. Paris: Dunod 1970

17. Greub, W., Halperin, S., Vanstone, R.: Connections, curvature, and cohomology. I. New York, NY: Academic Press 1972

18. Woodhouse, N.: Geometric quantization. Oxford: Oxford University Press 1980; Hurt, N.: Geometric quantization in action. Amsterdam: D. Reidel 1983

19. Stasheff, J.: Homological Reduction of Constrained Poisson Algebras, North Carolina Preprint, 1989

20. Śniatycki, J., Weinstein, A.: Lett. Math. Phys. 7, 155 (1983)

21. Arms, J., Marsden, J., Montcrief, V.: Commun. Math. Phys. 78, 455 (1981)

22. Fisch, J., Henneaux, M., Stasheff, J., Teitelboim, C.: Commun. Math. Phys. 120, 379 (1989)

Communicated by L. Alvarez-Gaumé 
